\title{
Guideline for the investigation and initial therapy of diarrhea-negative hemolytic uremic syndrome
}

\author{
Gema Ariceta • Nesrin Besbas • Sally Johnson • \\ Diana Karpman • Daniel Landau • Christoph Licht • \\ Chantal Loirat - Carmine Pecoraro • C. Mark Taylor • \\ Nicole Van de Kar • Johan VandeWalle • \\ Lothar B. Zimmerhackl • \\ The European Paediatric Study Group for HUS
}

Received: 11 January 2008 /Revised: 13 March 2008 / Accepted: 14 March 2008/ Published online: 18 September 2008

(C) IPNA 2008

\begin{abstract}
This guideline for the investigation and initial treatment of atypical hemolytic uremic syndrome (HUS) is intended to offer an approach based on opinion, as evidence is lacking. It builds on the current ability to identify the etiology of specific diagnostic sub-groups of HUS. HUS in children is mostly due to infection, enterohemorrhagic Escherichia coli (EHEC), Shigella dysenteriae type 1 in some geographic regions, and invasive Streptococcus pneumoniae. These sub-groups are relatively straightforward to diagnose. Their management, which is outside the remit
\end{abstract}

This article was drafted by S. Johnson, C. Loirat and C.M. Taylor

\section{G. Ariceta}

Hospital de Cruces,

Barakaldo,

Vizcaya, Spain

N. Besbas

Pediatric Nephrology Unit, Department of Pediatrics,

Faculty of Medicine,

Hacettepe University,

Ankara, Turkey

\section{S. Johnson $\cdot$ C. M. Taylor $(\bowtie)$}

Department of Nephrology,

Birmingham Children's Hospital,

Birmingham B4 6NH, UK

e-mail: cm.taylor@bch.nhs.uk

D. Karpman

Department of Paediatrics,

Clinical Sciences Lund University,

Lund, Sweden

D. Landau

Pediatric Nephrology,

Soroka Medical Center,

Beer Sheva, Israel of this guideline, is related to control of infection where that is necessary and supportive measures for the anemia and acute renal failure. A thorough investigation of the remainder of childhood HUS cases, commonly referred to as "atypical" HUS, will reveal a risk factor for the syndrome in approximately $60 \%$ of cases. Disorders of complement regulation are, numerically, the most important. The outcome for children with atypical HUS is poor, and, because of the rarity of these disorders, clinical experience is scanty. Some cases of complement dysfunc-

C. Licht

The Hospital for Sick Children, University of Toronto,

Toronto, Ontario, Canada

C. Loirat

Assistance Publique-Hopitaux de Paris, Universite Paris 7,

Service de Nephrologie, Hopital Robert Debre,

Paris, France

C. Pecoraro

Department of Nephrology and Urology,

Santobono Children's Hospital,

Naples, Italy

N. Van de Kar

University Medical Center St Radboud,

Nijmegen, The Netherlands

J. VandeWalle

UZG Pediatrics, University Hospital,

Gent, Belgium

L. B. Zimmerhack1

Universitäts-Klinik für Kinder-und Jugendheilkunde,

Medizinische Universität Innsbruck,

Innsbruck, Austria 
tion appear to respond to plasma therapy. The therapeutic part of this guideline is the consensus of the contributing authors and is based on limited information from uncontrolled studies. The guideline proposes urgent and empirical plasmapheresis replacement with whole plasma fraction for the first month after diagnosis. This should only be undertaken in specialized pediatric nephrology centers where appropriate medical and nursing skills are available. The guideline includes defined terminology and audit points so that the early clinical effectiveness of the strategy can be evaluated.

Keywords Hemolytic uremic syndrome - Atypical HUS . Thrombotic thrombocytopenic purpura . Enterohemorrhagic Escherichia coli . Complement factor $\mathrm{H}$ - Complement factor I - Complement factor B · Complement C3 . Membrane co-factor protein MCP (CD46) - A disintegrin and metalloproteinase with a thrombospondin type 1 motif, member 13 (ADAMTS13) · Plasmapheresis

\section{Purpose and scope of the guideline}

The guideline describes a clinical pathway for cases of hemolytic uremic syndrome (HUS) and is intended to offer an approach based on opinion, as evidence is lacking. It is designed to streamline the recognition of those cases of HUS that have etiologies other than enterohemorrhagic Escherichia coli (EHEC), Shigella dysenteriae type 1 or Streptococcus pneumoniae infection: commonly called "atypical" HUS. It addresses the question "How should I investigate and treat a new patient with atypical HUS?" It offers a standardized, comprehensive and evidence-based approach to investigation in order to maximize the ability to reach a sub-group diagnosis based on causation. The therapeutic component of the guideline is based on expert consensus, because evidence is lacking. It applies to the first month of treatment and standardizes the dosage of plasmapheresis. It defines clinical endpoints and indicates audit measures.

\section{Classification/terminology based on causation}

HUS is clinically recognized by the simultaneous occurrence of microangiopathic hemolytic anemia, thrombocytopenia (platelets typically $<150 \times 10^{9} / 1$ ) and acute renal failure.

The classification and terminology used in this guideline, "Classification of HUS, thrombotic thrombocytopenic purpura (TTP) and related disorders" is set out in detail in the report of the European Paediatric Research Group for HUS published in 2006 [1] and is summarized in Table 1.

The intention is to investigate patients appropriately so as to identify an etiologically based sub-group diagnosis wherever possible, i.e. Box 1 in the scheme tabled above. It is now
Table 1 Classification of HUS, TTP and related disorders (ADAMTS13 a disintegrin and metalloproteinase with a thrombospondin type 1 motif, member $13, H I V$ human immunodeficiency virus, HELLP hemolysis, elevated liver enzymes, low platelet count)

Box 1: etiology advanced

1.i. Infection-induced

(a) Shiga and shiga-like toxin-producing bacteria, Enterohemorrhagic Escherichia coli, Shigella dysenteriae type 1

(b) Streptococcus pneumoniae

1.ii. Disorders of complement regulation

(a) Genetic

(b) Acquired

1.iii. ADAMTS13 deficiency

(a) Genetic

(b) Acquired

1.iv. Defective cobalamin metabolism

1.v. Quinine-induced

Box 2: etiology unknown

2.i. HIV infection

2.ii. Malignancy, cancer chemotherapy, ionizing radiation

2.iii. Calcineurin inhibitors and transplantation

2.iv. Pregnancy HELLP syndrome, contraceptive pill

2.v. Systemic lupus erythematosus, anti-phospholipid antibody syndrome

2.vi. Glomerulopathy

2.vii. Familial not included in level 1

2.viii. Unclassified

recognized that the condition of some patients, that, hitherto, would have been described only by a clinical association (Box 2 ), can now be more precisely defined. Full investigation, sometimes done retrospectively, can move the diagnosis from the generally unsatisfactory level of clinical association to a meaningful etiology. Note that cases with a deficiency of a disintegrin and metalloproteinase with a thrombospondin type 1 motif, member 13 (ADAMTS13) (von Willebrand factor protease) may present initially as HUS, although this is rare, and they more usually have the phenotype of TTP. Patients with ADAMTS13 deficiency have an established therapy, which is outside the scope of this guideline [2].

\section{Guideline}

Step 1: screen new HUS patients according to clinical presentation

Figure 1 illustrates step 1 of the guideline. Following the recognition of HUS, cases should be assigned to one of the three clinically recognizable patterns of presentation indicated in Fig. 1:

(a) Children older than 6 months presenting with diarrhea or bloody diarrhea. These require investigation to determine the cause of gastrointestinal infection, using methods that are relevant to the EHEC or Shigella dysenteriae type 1 of the local region. 
Fig. 1 Step 1 of the guideline. Recognition of atypical HUS. Superscript numbers refer to notes to step 1

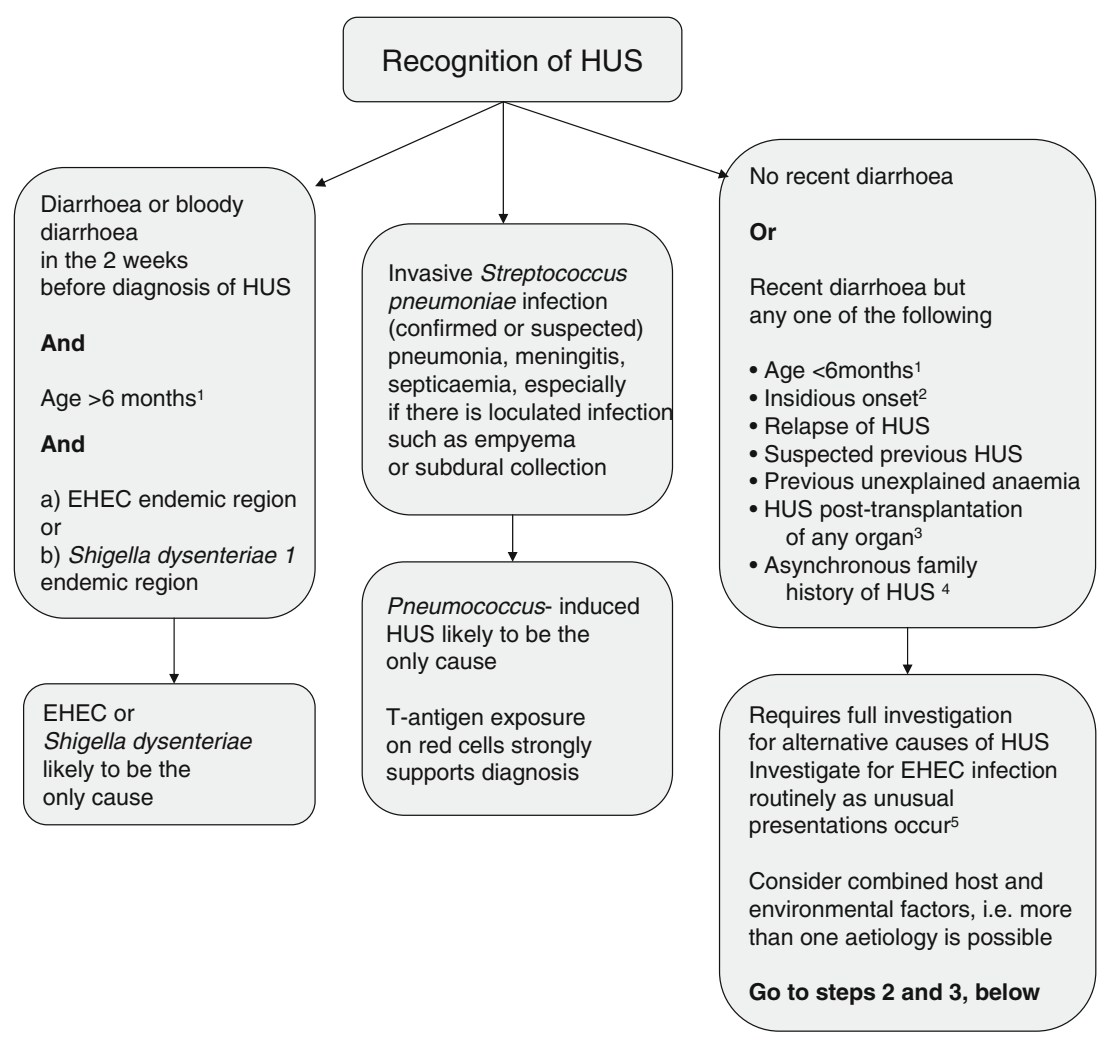

(b) Children with suspected invasive pneumococcal infection. Bacteriological confirmation should be sought. Exposure of the Thomsen-Friedenreich antigen on red blood cells strongly supports the diagnosis [3].

(c) All other cases can be regarded as atypical and require full investigation according to step 2 .

\section{Notes to step 1}

1. The cut-off point of 6 months is designed to help the positive selection of patients with EHEC from other causes of HUS. It is, to some extent, arbitrary, and the power to select varies in different populations according to the local epidemiology of EHEC infection. In central Europe $5 \%$ of EHEC-induced HUS occurs in infants below 6 months of age [4], while, in the UK, this is $<3 \%$ [5]. Although HUS induced by disorders of complement regulation can arise at any age, many cases present in the first few months of life. In addition, exposure to EHEC is less likely in this age group (preweaning), and, therefore, suspicion of other causes of HUS in those presenting under 6 months of age should be high. Between 1 year and 5 years of age, the incidence of EHEC-induced HUS exceeds all other causes by approximately 10:1 [5].

2. HUS induced by EHEC, Shigella dysenteriae or Streptococcus pneumoniae is rapid in onset. Anemia, thrombocytopenia and oliguric renal failure usually become apparent over a few days. HUS induced by complement dysregulation or ADAMTS13 deficiency can also be abrupt. However, an insidious onset over more than 10 days, fluctuating clinical signs and laboratory parameters and marked hypertension increase the likelihood of a non-infective cause [6].

3. HUS can follow transplantation of other organs as well as the kidney [7, 8]. The role of drugs, especially calcineurin inhibitors, has been suspected but not proven [9]. HUS in a kidney-transplant patient raises the question of a host risk factor, whether or not the original cause of the patient's end-stage renal failure was known to be HUS.

4. Two patterns of familial HUS occur. In an outbreak of EHEC infection family members may develop HUS, either simultaneously (common source of infection) or a few weeks apart (secondary spread). These families do not require investigation beyond confirmation of the EHEC infection. By contrast, families with asynchronous HUS are very likely to have inherited risk factors and require full investigation.

5. EHEC infection can cause HUS without diarrhea [4]. Also, EHEC urinary tract infection can induce HUS [10]. Always use microscopy and culture a urine sample. Use locally developed public health/microbiological services to identify the EHEC. This may include stool culture, \pm enhancement and selection techniques, \pm gene probes for verocytotoxin (VT) subtypes, serotyping of 
the identified coliform organism, serological response to locally relevant $\mathrm{O}$ serotypes $[11,12]$.

Step 2: investigate according to the recommendations in Table 2

The condition of children identified as having atypical HUS in step 1 should be fully investigated, using the comprehensive list of tests in step 2 of the guideline (Table 2). Because EHEC-induced HUS is common and unusual presentations can occur, it is important to conduct locally relevant investigations for EHEC in addition to those in Table 2 (see Fig. 1, note 5). For example, EHEC infection in the urinary tract occurs rarely and should be considered. If Escherichia coli is cultured from a urine sample it should be investigated for EHEC properties. If EHEC infection is confirmed in a patient who presented without diarrhea, the probability of a co-existing inherited risk factor such as complement dysregulation is much reduced, but not negated. Step 2 of the guideline is recommended.

Some of the investigations in Table 2 are highly specialized and, until recently, were undertaken only as part of research activity. Increasingly, they are being offered as an accredited diagnostic service. A list of laboratories that provide diagnostic assistance is given in Appendix 1.

\section{Notes to step 2}

1. If C3 level is low, this indicates complement dysregulation, but C3 levels may be normal in patients with

Table 2 Step 2 of the guideline. Recommended list of investigations for patients identified as having atypical HUS in step 1. Superscript numbers refer to the notes to step 2. ADAMTS13 a disintegrin and metalloproteinase with a thrombospondin type 1 motif, member 13 , $v W F c p$ von Willebrand factor cleaving protease, HIV human immu- dysregulation. A normal result does not exclude a complement disorder [13].

2. Factor $\mathrm{H}$ and factor I plasma concentrations may be normal in cases with mutations [13].

3. Mutation analysis of all the above factors should be undertaken, irrespective of $\mathrm{C} 3$ or factor $\mathrm{H}$ levels, particularly before transplantation is considered. A list of laboratories with special service or research interest in complement regulation and their contact details appears in the appendix.

4. ADAMTS13 deficiency generally does not present as HUS but as thrombotic thrombocytopenic purpura (TTP). Congenital deficiency, Upshaw-Schulmann syndrome, occurs either in the neonatal period or later in childhood, and an acquired form due to anti-ADAMTS13 antibodies occurs in adolescence. Since the condition of these patients may present as HUS, investigation of ADAMTS13 activity is indicated. A list of laboratories with special service or research interest in ADAMTS13 appears in the appendix. The further management of these cases lies outside the remit of this guideline.

\section{Step 3: treatment}

This step, outlined in Fig. 2, makes recommendations for the treatment of patients identified as having atypical HUS from step 1. It relies on the observation that disorders of complement regulation are numerically the most likely etiologies [1]. Expert consensus holds that most patients in this group benefit from plasmapheresis. Because it takes nodeficiency virus, HELLP hemolysis, elevated liver enzymes, low platelet count, $M C P$ membrane co-factor protein, $F A C S$ fluorescenceactivated cell sorter, MMACHC methylmalonic aciduria and homocystinuria type $\mathrm{C}$ protein

Classification (see boxes 1 and 2) Investigation

1.ii. Disorders of complement regulation

C3 (plasma/serum) ${ }^{1}$

Factor $\mathrm{H}$ and factor I concentration (plasma/serum) ${ }^{2}$

Factor $\mathrm{H}$ autoantibody

MCP (CD46) (surface expression on mononuclear leukocytes by FACS)

Gene mutation analysis in factor $\mathrm{H}$, factor I, MCP, factor B and $\mathrm{C}^{3}$

1.iii. ADAMTS13 (vWFcp) deficiency

inherited or acquired classification

Plasma vWF protease (ADAMTS13) activity \pm inhibitor (plasma) ${ }^{4}$. Measure in acute phase of illness. Significant if activity $<10 \%$ of normal. If low, check for autoantibody inhibitor. Repeat in remission. If persistently low activity in absence of inhibitor, inherited deficiency likely. Genetic confirmation optional at specialized genetic laboratories

1.iv. Cobalamin metabolism

Homocysteine, methylmalonic acid (plasma and urine) \pm mutation analysis in MMACHC gene

2.i. HIV

Serology

2.iv. Pregnancy HELLP syndrome

Pregnancy test, liver enzymes. Always consider pregnancy in teenage girl with HUS or TTP. Investigate as in 1.ii. and 1.iii. above

2.v. Miscellaneous

Antinuclear antibody, lupus anticoagulant, anti-phospholipid antibodies 
Fig. 2 Step 3 of the guideline. Recommendations for the treatment of patients identified as having atypical HUS from step 1. Superscript numbers refer to notes to step 3

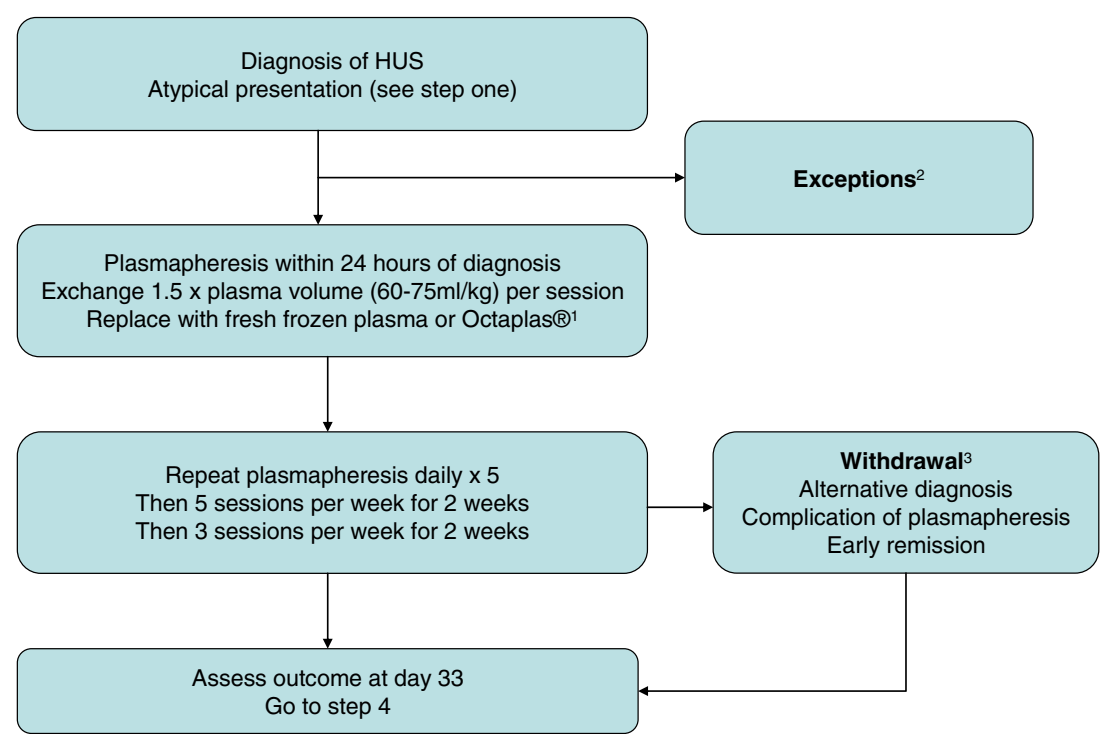

time to complete the investigations that lead to a diagnostic sub-group, initial treatment is necessarily empirical. Moreover, because atypical HUS is a destructive disorder, and up to a quarter of children progress to end-stage renal failure in their first episode [14, 15], the guideline indicates that treatment should be started urgently, that is to say, within 24 hours of diagnosis of atypical HUS. This step does not apply to patients in whom EHEC infection is confirmed.

\section{Notes to step 3}

1. Plasmapheresis is a highly technical and complicated treatment. Vascular access and plasma exchange should only be undertaken in a specialized pediatric environment by staff competent with the procedures [16]. Plasma exchange may be by plasma filtration or centrifugal separator, according to local practice and expertise, and should use local practice for anticoagulation of the extracorporeal circuit. The first plasma exchange should be instituted as soon as it is practicable after the diagnosis of atypical HUS, and once the child's condition has been stabilized with regard to hydration, electrolyte disturbance, anemia and blood pressure.

Exchange 1.5-times the expected plasma volume, which is equivalent to between $60 \mathrm{ml} / \mathrm{kg}$ body weight and $75 \mathrm{ml} / \mathrm{kg}$ body weight. Replace plasma with whole plasma fraction, either virus-inactivated pooled plasma (e.g. Octaplas ${ }^{\circledR}$ ) or individual units of fresh frozen plasma from screened donors [17]. The risk of transmission of viruses and prions can be reduced by the choice of plasma source, but it cannot be eliminated, and patients and parents must be fully informed of the potential risks and benefits. Plasma exchange should be performed daily for 5 days, then five times a week for
2 weeks, and then three times a week for 2 weeks. The dosage, frequency and duration of plasmapheresis are all arbitrary, but are influenced by the experience of the authors and rare published cases $[18,19]$.

2. Exceptions. There are some occasions when clinicians may elect not to use this empirical guideline.

(a) where the effect of an alternative treatment can be anticipated. For example, HUS in a sibling of a patient with congenital ADAMTS13 deficiency is likely to have the same diagnosis and might be expected to respond to plasma infusion $10 \mathrm{ml} / \mathrm{kg}$ per day.

(b) where the clinical presentation strongly suggests early onset cobalamin-C disorder (feeding difficulty, failure to thrive, hypotonia, lethargy, leukopenia and megaloblastic anemia).

(c) where there are technical difficulties in achieving vascular access in small children.

(d) where the clinician considers that the risks of plasmapheresis outweigh the benefits in a child with apparently mild renal involvement and conserved urine output, in which case the decision might be deferred. However, clinicians should be aware that thrombotic microangiopathy is a destructive process, and that, in the few reported patients with factor $\mathrm{H}$ mutations in whom plasma therapy appeared successful, treatment had been started before there was renal impairment [19-22].

3. Withdrawal from the guideline. Patients can be withdrawn from the intensive plasma exchange regime before 1 month on the advice of the local physician if:

(a) an alternative diagnosis is reached where the condition is not expected to respond to plasma therapy, for example cobalamin-C disorder. 
(b) a diagnosis of congenital ADAMTS13 deficiency is made, in which case guidelines for TTP should be followed [2].

(c) there are complications or side effects of plasma exchange that necessitate the withdrawal of treatment.

(d) the patient enters a hematological remission (see below). In this case ongoing treatment needs to be planned in the knowledge of the cause of HUS.

Step 4: defining patient outcome

The end-point for determining early outcome has been set arbitrarily at 1 month (to be precise day 33). The guideline does not address ongoing active treatment in patients who appear to have remission from their disease, nor does it advise on what action to take in patients who have failed to respond at the end of the first month. The authors recognize that, for some patients, the etiological sub-group diagnosis may not be available at that time.

We propose that a clinical record of each patient's outcome be made, using the following terminology:

Hematological remission is defined as a platelet count $>150 \times 10^{9} / 1$ for 2 weeks and no signs of hemolysis [fragmented red cells, elevated lactic dehydrogenase (LDH) level]. This definition is independent of renal function.

Hematological relapse of HUS is defined as a return of microangiopathic hemolytic anemia and thrombocytopenia after these parameters have normalized for a period of a least 2 weeks.

Renal function is graded under three levels for simplicity: renal failure requiring dialysis, renal impairment, in which there is independent kidney function but plasma creatinine level is elevated for age or glomerular filtration rate (GFR) $<80 \mathrm{ml} / \mathrm{min}$ per $1.73 \mathrm{~m}^{2}$ body surface area (BSA) by the Schwartz formula or a formal clearance assay, and normal in which plasma creatinine level is appropriate for age or GFR $\geq 80 \mathrm{ml} / \mathrm{min}$ per $1.73 \mathrm{~m}^{2}$ BSA.

Proteinuria should be assessed as a numerical variable, protein/creatinine ratio on first-voided urine sample in the morning.

Blood pressure should be assessed as a numerical variable and expressed as a centile factored for age and gender [23, 24]. In addition, if antihypertensive therapy is used, the number of different antihypertensive drugs should be stated.

\section{Step 5: audit}

Because of the rarity of these diseases, audit can only be undertaken on a multi-center, national or international basis. For this to happen there needs to be a standardized approach to management and a common terminology to describe diagnostic sub-groups and their outcomes. With this in place, audit should be capable of clarifying the expected outcome of children with atypical HUS, a necessary basis on which to design future therapeutic trials.

Audit should address the following points

(1) What proportion of cases of atypical HUS cases are fully investigated according to the guideline, and what is the yield of that investigation?

(2) What proportion of patients are treated according to the guideline? How many meet the criteria for exception or are withdrawn from plasma exchange in the first month, and why?

(3) What proportion of patients have entered hematological remission by the end of the first month, and what proportions have preservation or loss of renal function?

(4) What is the expected 1-month outcome for patients with specific sub-group diagnoses?

Various registries exist that collate etiological and outcome data of children with atypical HUS. Every attempt should be made to invite patients to participate in such registries. Registries should be prepared to collaborate, to expedite answers to the audit questions outlined above. Information about current registries is given in Appendix 2

\section{Discussion}

Until recently, it was not possible for clinicians to identify the cause of atypical HUS unless supported by a research laboratory. Today, specialized tests, including genetic analyses, are being rolled out as diagnostic services, and, although they are expensive, it is feasible and necessary that patients be fully investigated according to the plan described in step 2. Evidence from disease registries indicates that a risk factor or sub-group diagnosis will be identified in approximately $50 \%$ of cases not associated with diarrhea, or enterohemorrhagic E. coli, or invasive pneumococcal infection $[14,15]$.

The benefit to patients of obtaining an etiological diagnosis is expected to translate into better clinical advice and treatment, although the evidence for this, at present, is theoretical, anecdotal or lacking (see below). Moreover, in the first weeks after presentation, clinicians usually will not have the results of definitive investigations such as genetic tests, but plasma concentrations of complement factors and ADAMTS13 activity are likely to be available sooner. Clinicians, therefore, have to decide empirically about initial treatment, and there is currently no standardized approach.

There is a precedent for an empirical approach. It is widely quoted that the mortality of TTP has been reduced from $90 \%$ to approximately $10 \%$ with plasma therapy [25]. Only later was this given theoretical justification with our 
understanding of the role of ADAMTS13 deficiency in the pathogenesis of TTP.

Complement regulatory abnormalities, either inherited or acquired through autoantibodies, are frequent causes of atypical HUS [14]. The historic rates of mortality and morbidity of this group are high [6]. Anecdotally, prompt plasma exchange or plasma infusion has been associated with remission [19-22, 26-29]. Specifically for factor H-related cases, there are reports of five children with homozygous or compound heterozygous deficiency in whom the effects of plasma infusion have been described [21, 22, 26, 27, 29, 30]. All showed early benefit, with a plasma dose range of $12-$ $20 \mathrm{ml} / \mathrm{kg}$ body weight given up to three times per week. However, the disease in one child became refractory to this regime [27]. It should also be noted that repeated plasma infusion is limited for patients who are oligoanuric, because of the risk of volume overload. There are three patients with heterozygous factor $\mathrm{H}$ mutations for whom the effects of plasma therapy have been described $[19,20]$. One, treated with regular plasma infusion, developed hyperproteinemia necessitating plasma exchange. Another received regular plasma exchange after transplantation and maintained graft function in spite of HUS recurrences. Davin et al. [19] reported monozygous twins who had responded to plasma exchange, but the first twin, who was reduced to regular plasma infusion, progressed to end-stage renal failure. The second twin, maintained on plasma exchange every 2 weeks, retained normal function. Although there are data on the effects of plasma therapy in two retrospective patient cohorts, one of which includes adults, there is insufficient detail of the plasma regime for conclusions to be drawn about benefit $[14,15]$.

The rationale for plasma treatment is to replace absent or mutated circulating complement regulators, such as factor $\mathrm{H}$. However, the pathogenesis of HUS induced by factor $\mathrm{H}$ mutation is incompletely understood. Many mutations are heterozygous, suggesting either a dominant negative effect or haplotype insufficiency. Plasma infusion is likely to overcome the latter but not the former. The dose of factor $\mathrm{H}$ needed to correct complement regulation where it matters, presumably on endothelial surfaces in the kidney, is unknown. The halflife of factor $\mathrm{H}$ in plasma is approximately 6 days [21], but this may not be relevant in the accelerated site-specific complement activation that underlies HUS.

Patients with isolated MCP (CD46) dysfunction do not appear to benefit from plasma therapy $[14,15]$. This may be because MCP is a membrane-bound complement regulator, not a circulating one, and there is a high spontaneous remission rate. Little is known about the role of plasma therapy for other forms of complement dysregulation. HUS has been reported in patients with autoantibodies to factor $\mathrm{H}$ [31]. In theory, plasma exchange might be expected to remove the autoantibody and provide additional factor $\mathrm{H}$.
Similarly, mutated factor B that permits excessive complement activation [32] might be removed by plasma exchange.

We estimate that, in order for one patient in group 1.ii. (disorders of complement dysregulation) to be treated with what is proposed to be an effective treatment, on average 12 patients will be treated who do not have these diagnoses or might be expected not to benefit.

Plasmapheresis is not without risk [16]. The justification for urgent treatment at a stage when the etiological diagnosis is unknown can only be made on the high risk of early death, irreversible kidney damage or risks associated with prolonged disease activity (dialysis, blood transfusion, etc.) in this group as a whole $[14,15]$. Given that contemporary patients are usually offered plasma therapy of various doses and duration, the natural outcome of untreated cases is unknown. Historic controls may not be informative because of improvements in the general supportive care of children with acute renal failure.

The therapeutic recommendation of this guideline is, therefore, a balanced judgment that relies heavily on the experience of the authors. It is not a research proposal, as there is no equipoise for a comparative treatment. However, a standardized approach to diagnosis and management lends itself to prospective audit, which, in the authors' view, is obligatory. There are various national and international registries of HUS that are capable of undertaking this. Standardization now is important, because alternative treatments, such as complement factor $\mathrm{H}$ concentrate, synthetic complement regulators and antibodies against complement effector proteins such as $\mathrm{C} 5 \mathrm{a}$, are likely to be available in the near future and will need to be tested against a standard [33]. This guideline should, therefore, serve for only a limited time, probably not more than 3 years from publication.

Treatment beyond the first month from presentation is outside the remit of the guideline.

\section{Appendices}

Appendix 1. Laboratories providing specialized investigation

Note, clinicians requiring to know the accreditation status of any of the following laboratories should obtain current information from the contacts named below.

\section{Complement studies}

Service d'Immunologie Biologique, Hopital Europeén George Pompidou, 20-40 rue Leblanc, 75908 Paris cedex 15, France.

An expert complement laboratory that undertakes the measurement of factor $\mathrm{H}$, factor I, factor $\mathrm{B}$, MCP surface 
expression on mononuclear leukocytes, anti-factor $\mathrm{H}$ antibodies. Results provided within 1 week. In addition, full mutation analyses of factor $\mathrm{H}$, factor I, MCP, factor B and C3 are performed. Results of genetic screening provided within 3 months. There is a charge for this.

For advice contact Dr. Veronique Fremeaux-Bacchi; Email: veronique.fremeaux-bacchi@egp.aphp.fr

The Northern Genetics Service, Institute of Human Genetics, Newcastle upon Tyne Hospitals NHS Foundation Trust, International Centre for Life, Newcastle upon Tyne, NE1 3BZ, UK.

An accredited genetic diagnostic service that provides mutation screening of factor $\mathrm{H}$, factor I and MCP, as well as identification of the $C F H:$ :CFHRI hybrid and copy number of CFHR1 and CFHR3. Serum levels of C3, C4, factor $\mathrm{H}$ and factor I are measured simultaneously in an accredited diagnostic immunology laboratory. There is a charge for both services. The service is linked to the Institute of Human Genetics, University of Newcastle, which is active in researching the genetic aspects of complement regulation in HUS.

For advice contact Prof. Tim Goodship; Email: t.h.j. goodship@ncl.ac.uk

For the service laboratory, Email: lisa.strain@nuth.nhs.uk

Mario Negri Institute for Pharmacological Research, Via Gavazzeni 11, 24125 Bergamo, Italy.

A research active group that undertakes protein and genetic investigation of complement factors $\mathrm{H}$, I and $\mathrm{B}, \mathrm{C} 3$ and MCP (CD46), as well as VWF protease (ADAMTS13). Results provided within 4 months. There is no charge for these tests. Clinicians must supply full clinical details, using the institute's case questionnaire for inclusion in the department's clinical database.

For advice contact Prof. Giuseppe Remuzzi; Email: gremuzzi@marionegri.it and Dr. Marina Noris; Email: noris@marionegri.it

Department of Biological Infection, Leibniz Institute for Natural Product Research and Infection Biology, Hans Knoell Institute for Natural Product Research, Beutenbergstr. 11a, 07745 Jena, Germany.

Complement protein analysis and genomic investigation of complement factor $\mathrm{H}$, factor I and MCP (CD46) is available as a service from the research group headed by Prof. Peter Zipfel. There is a charge for this service; scientific aspects may have exemption.

Contact Prof. Peter Zipfel; Email peter.zipfel@hki-jena.de

Department of Pediatrics, BMC C14, Lund University, Klinikgatan 28, 22184 Lund, Sweden

Protein and genetic investigation of complement factors $\mathrm{H}$ and I and MCP, as well as vWF cleaving protease ADAMTS13. Investigation of patients in northern Europe and Scandinavia. There is a charge for this service. Results provided within 3 months.
For advice contact: Prof. Diana Karpman; Email diana. karpman@med.lu.se

Laboratory for Paediatrics and Neurology, Radboud University Nijmegen Medical Center, Postbus 9101, 6500 HB Nijmegen, Geert Grooteplein 106525 GA Nijmegen, The Netherlands.

A genetic service is offered for mutational analysis of factor $\mathrm{H}$, factor $\mathrm{I}, \mathrm{MCP}$ (CD46) factor $\mathrm{B}, \mathrm{C} 3$ and ADAMTS13. The investigations are performed on genomic DNA by a combination of polymerase chain reaction (PCR) and sequence analysis. Results will be delivered within 6 months. There is a charge. Clinicians are asked to supply clinical details of patients.

For advice contact Dr. L.P. van den Heuvel; Email: B. vandenHeuvel@cukz.umcn.nl

Immunology Unit and Research Unit, Hospital Universitario La Paz, Paseo de la Castellana, 261, 28046 Madrid, Spain and Laboratory of Complement Genetics, Centro de Investigaciones Biológicas, Ramiro de Maeztu 9, 28040Madrid, Spain.

These are research laboratories expert in complement genetics and functional analysis relevant to HUS. They offer measurements of $\mathrm{C} 3$, factor $\mathrm{H}$, factor $\mathrm{I}$, factor $\mathrm{B}, \mathrm{MCP}$ (on peripheral blood leukocytes), anti-factor $\mathrm{H}$ antibodies and functional assays for factor $\mathrm{H}$. They also provide services for the mutational analysis of $C F H, C F I, M C P(C D 46), C F B$ and $C 3$; for the genotyping of polymorphisms associated with increased HUS risk in the $C F H$ and $M C P$ genes; for the identification of $C F H:: C F H R 1$ hybrid genes, and they analyze for the CFHR1-CFHR3 deletion. Results are provided within 2 months. There is a charge for these services. Clinicians are asked to supply clinical details of patients.

For advice contact Prof. Santiago Rodríguez de Córdoba; Email: srdecordoba@cib.csic.es

Dr. Pilar Sánchez-Corral; Email: psanchez.hulp@salud. madrid.org

von Willebrand Protease

U770 INSERM, Hopital de Bicetre, 80 rue du General Leclerc, 94276 Le Kremlin-Bicetre cedex, France.

The assay for specific protease activity is technically demanding but available in coagulation laboratories in several countries. However, a dependable service can be obtained from INSERM above. Charges may apply.

For advice contact Dr. Agnes Veyradier; Email agnes. veyradier@abc.aphp.fr

Haemostasis Research Unit, Haematology Department, University College London, 1st floor, 51 Chenies Mews, London WC1E 6HX, UK

Expert laboratory with ability to identify protease activity and the presence of inhibitors. Charges apply for diagnostic tests outside agreed research projects.

Sample requirement: either citrated blood shipped immediately, or double spin at 2,000 $g$ and divide plasma 
into aliquots (minimum plasma for assay $2 \times 0.5 \mathrm{ml}$ ), freeze at $-70^{\circ} \mathrm{C}$ and send on dry ice. Samples must arrive before $5 \mathrm{pm}$ Monday-Friday (avoid public holidays!) with a request form available from either Dr. Ian Mackie or Gordon Purdy. Phone +44-20-7679 6416/6423 before sending sample to ensure that it is processed. E-mail: i. Mackie@ucl.ac.uk or g.purdy@ucl.ac.uk

University Medical Center Hamburg-Eppendorf, Department of Pediatric Hematology and Oncology, Molecular Genetics Laboratory, Martinistrasse 52, 20246, Hamburg, Germany

The laboratory provides molecular genetic investigation of ADAMTS13 on a routine basis. Results are usually available within 2 months. Genetic studies on von Willebrand factor are also performed to identify possible "ADAMTS13 resistance" mutations of VWF in particular cases. New mutations are expressed on a research basis. Charges apply, but at a reduced rate for institutions. ADAMTS13 activity testing is available by the fluorescence resonance-energy transfer (FRET) assay.

For advice contact Prof. Dr. Reinhard Schneppenheim; Email: schneppenheim@uke.de or Florian Oyen: Email: f. oyen@uke.de

\section{Appendix 2 Current registries for atypical HUS}

The website of the European Paediatric Study Group for HUS provides a list on national contacts for European countries. See http://www.espn.cardiff.ac.uk/registries

International Registry and Biorepository for TMA.

Schneider Children's Hospital of the North Shore-LIJ Health System, New Hyde Park, New York 11040, USA

Coordinator, Catherine Hoffman; Email: choffman@nshs.edu

Principal Investigator, Howard Trachtman; Email: trachtma@lij.edu

The Innsbruck registry for HUS (Austria, Germany, Switzerland, Czech Republic, Slovakia, Slovenia and Hungary) can be contacted through http://www.hemolyticuremic-syndrome.org

\section{References}

1. Besbas N, Karpman D, Landau D, Loirat C, Proesmans W, Remuzzi G, Rizzoni G, Taylor CM, Van de Kar N, Zimmerhackl LB, European Paediatric Research Group for HUS (2006) A classification of hemolytic uremic syndrome and thrombotic thrombocytopenic purpura and related disorders. Kidney Int 70:423-431

2. Allford SL, Hunt BJ, Rose P, Machin SJ, Haemostasis and Thrombosis Task Force, British Committee for Standards in Haematology (2003) Guidelines on the diagnosis and management of the thrombotic microangiopathic haemolytic anaemias. $\mathrm{Br}$ J Haematol 120:556-573
3. Novak RW, Martin CR, Orsini EN (1983) Hemolytic-uremic syndrome and T-cryptantigen exposure by neuraminidaseproducing pneumococci: an emerging problem? Pediatr Pathol $1: 409-413$

4. Gerber A, Karch H, Allerberger F, Verweyen HM, Zimmerhackl LB (2002) Clinical course and the role of shiga toxin-producing Escherichia coli infection in the hemolytic-uremic syndrome in pediatric patients, 1997-2000, in Germany and Austria: a prospective study. J Infect Dis 186:493-500

5. Milford DV, Taylor CM, Guttridge B, Hall SM, Rowe B, Kleanthous H (1990) Haemolytic uraemic syndromes in the British Isles 1985-8: association with verocytotoxin producing Escherichia coli. Part 1: clinical and epidemiological aspects. Arch Dis Child 65:716-721

6. Fitzpatrick MM, Walters MD, Trompeter RS, Dillon MJ, Barratt TM (1993) Atypical (non-diarrhea-associated) hemolytic-uremic syndrome in childhood. J Pediatr 122:532-537

7. Bonser RS, Adu D, Franklin I, McMaster P (1984) Cyclosporininduced haemolytic uraemic syndrome in liver allograft recipient. Lancet 2:1337

8. Moake JL, Byrnes JJ (1996) Thrombotic microangiopathies associated with drugs and bone marrow transplantation. Hematol Oncol Clin North Am 10:485-497

9. Abraham KA, Little MA, Dorman AM, Walshe JJ (2000) Hemolytic-uremic syndrome in association with both cyclosporine and tacrolimus. Transpl Int 13:443-447

10. Hogan MC, Gloor JM, Uhl JR, Cockerill FR, Milliner DS (2001) Two cases of non-O157:H7 Escherichia coli hemolytic uremic syndrome caused by urinary tract infection. Am J Kidney Dis 38:E22

11. Bielaszewska M (2007) Shiga toxin-mediated hemolytic uremic syndrome: time to change the diagnostic paradigm? PLoS ONE 2:e1024

12. Pradel N, Livrelli V, De Champs C, Palcoux JB, Reynaud A, Scheutz F, Sirot J, Joly B, Forestier C (2000) Prevalence and characterization of Shiga toxin-producing Escherichia coli isolated from cattle, food, and children during a one-year prospective study in France. J Clin Microbiol 38:1023-1031

13. Saunders RE, Goodship TH, Zipfel PF, Perkins SJ (2006) An interactive web database of factor $\mathrm{H}$-associated hemolytic uremic syndrome mutations: insights into the structural consequences of disease-associated mutations. Hum Mutat 27:21-30

14. Sellier-Leclerc AL, Fremeaux-Bacchi V, Dragon-Durey MA, Macher MA, Niaudet P, Guest G, Boudailliez B, Bouissou F, Deschenes G, Gie S, Tsimaratos M, Fischbach M, Morin D, Nivet H, Alberti C, Loirat C, French Society of Pediatric Nephrology (2007) Differential impact of complement mutations on clinical characteristics in atypical hemolytic uremic syndrome. J Am Soc Nephrol 18:2392-2400

15. Caprioli J, Noris M, Brioschi S, Pianetti G, Castelletti F, Bettinaglio P, Mele C, Bresin E, Cassis L, Gamba S, Porrati F, Bucchioni S, Monteferrante G, Fang CJ, Liszewski MK, Kavanagh D, Atkinson JP, Remuzzi G, International Registry of Recurrent and Familial HUS/TTP (2006) Genetics of HUS: the impact of MCP, $\mathrm{CFH}$, and IF mutations on clinical presentation, response to treatment, and outcome. Blood 108:1267-1279

16. Michon B, Moghrabi A, Winikoff R, Barrette S, Bernstein ML, Champagne J, David M, Duval M, Hume HA, Robitaille N, Bélisle A, Champagne MA (2007) Complications of apheresis in children. Transfusion 47:1837-1842

17. O’Shaughnessy DF, Atterbury C, Bolton Maggs P, Murphy M, Thomas D, Yates S, Williamson LM, British Committee for Standards in Haematology, Blood Transfusion Task Force (2004) Guidelines for the use of fresh-frozen plasma, cryoprecipitate and cryosupernatant. Br J Haematol 126:11-28

18. Stratton JD, Warwicker P (2002) Successful treatment of factor Hrelated haemolytic uraemic syndrome. Nephrol Dial Transplant 17:684-685 
19. Davin JC, Olie KH, Verlaak R, Horuz F, Florquin S, Weening JJ, Groothoff JW, Strain L, Goodship TH (2006) Complement factor $\mathrm{H}$-associated atypical hemolytic uremic syndrome in monozygotic twins: concordant presentation, discordant response to treatment. Am J Kidney Dis 47:e27-e30

20. Filler G, Radhakrishnan S, Strain L, Hill A, Knoll G, Goodship TH (2004) Challenges in the management of infantile factor $\mathrm{H}$ associated hemolytic uremic syndrome. Pediatr Nephrol 19:908-911

21. Licht C, Weyersberg A, Heinen S, Stapenhorst L, Devenge J, Beck B, Waldherr R, Kirschfink M, Zipfel PF, Hoppe B (2005) Successful plasma therapy for atypical hemolytic uremic syndrome caused by factor $\mathrm{H}$ deficiency owing to a novel mutation in the complement cofactor protein domain 15. Am J Kidney Dis 45:415-421

22. Nathanson S, Fremeaux-Bacchi V, Deschenes G (2001) Successful plasma therapy in hemolytic uremic syndrome with factor $\mathrm{H}$ deficiency. Pediatr Nephrol 16:554-556

23. Jackson LV, Thalange NK, Cole TJ (2007) Blood pressure centiles for Great Britain. Arch Dis Child 92:298-303

24. National High Blood Pressure Education Program Working Group on Hypertension Control in Children and Adolescents (1996) Update on the 1987 Task Force report on high blood pressure in children and adolescents: a working group report from the National High Blood Pressure Education Program. Pediatrics 98:649-658

25. Rock GMD (2005) The management of thrombotic thrombocytopenic purpura in 2005. Semin Thromb Hemost 31:709-716

26. Landau D, Shalev H, Levy-Finer G, Polonsky A, Segev Y, Katchko L (2001) Familial hemolytic uremic syndrome asso- ciated with complement factor H deficiency. J Pediatr 138: 412-417

27. Nathanson S, Ulinski T, Frémeaux-Bacchi V, Deschênes G (2006) Secondary failure of plasma therapy in factor $\mathrm{H}$ deficiency. Pediatr Nephrol 21:1769-1771

28. Zurowska A, Zaluska-Lesniewska I, Hladny-Czerska W (2006) Successful prophylactic plasma infusions in recurrent atypical hemolytic-uremic syndrome. Przegl Lek 63 [Suppl 3]:223-225

29. Cho HY, Lee BS, Moon KC, Ha IS, Cheong HI, Choi Y (2007) Complete factor $\mathrm{H}$ deficiency-associated atypical hemolytic uremic syndrome in a neonate. Pediatr Nephrol 22:874880

30. Gerber A, Kirchhoff-Moradpour AH, Obieglo S, Brandis M, Kirschfink M, Zipfel PF, Goodship JA, Zimmerhack1 LB (2003) Successful (?) therapy of hemolytic-uremic syndrome with factor H abnormality. Pediatr Nephrol 18:952-955

31. Dragon-Durey MA, Loirat C, Cloarec S, Macher MA, Blouin J, Nivet H, Weiss L, Fridman WH, Frémeaux-Bacchi V (2005) Antifactor $\mathrm{H}$ autoantibodies associated with atypical hemolytic uremic syndrome. J Am Soc Nephrol 16:555-563

32. Goicoechea de Jorge E, Harris CL, Esparza-Gordillo J, Carreras L, Arranz EA, Garrido CA, López-Trascasa M, Sánchez-Corral P, Morgan BP, Rodríguez de Córdoba S (2007) Gain-of-function mutations in complement factor B are associated with atypical hemolytic uremic syndrome. Proc Natl Acad Sci USA 104:240-245

33. Jokiranta TS, Zipfel PF, Fremeaux-Bacchi V, Taylor CM, Goodship TJ, Noris M (2007) Where next with atypical hemolytic uremic syndrome? Mol Immunol 44:3889-3900 